\title{
In-situ local strain measurement in textile composites with embedded optical fibre sensors
}

\author{
S. Daggumati, E. Voet, I. De Baere, W. Van Paepegem \& J. Degrieck \\ Ghent University, Department of Materials Science and Engineering, Technologiepark-Zwijnaarde 903, 9052 \\ Zwijnaarde, Belgium
}

J. Xu, S. Lomov \& I. Verpoest

Katholieke Universiteit Leuven, Department of Metallurgy and Materials Engineering, Kasteelpark Arenberg 44, B-3001 Leuven, Belgium

\begin{abstract}
To understand the local strains inside a textile composite, numerical simulations are typically done on the scale of one repetitive unit cell of the weaving pattern. Periodic boundary conditions are applied to the edges of the unit cell and different load cases can then be applied to the unit cell of the textile composite. Most often, the periodic boundary conditions are applied on all faces of the unit cell, which implies the assumption that the material is repeating itself over an infinite distance in all three orthogonal directions. This assumption is more or less valid for the textile composite material in the midplane of thick laminates, where it is constrained by neighbouring material in all three directions. It is very difficult to validate such simulations, because local strain measurements inside a textile composite have rarely been done, and the interpretation is not straightforward. This paper shows the successful use of embedded optical fibre sensors to measure the local strains inside a satin weave carbon/PPS composite (typically used in aerospace applications). The length of the Bragg grating inside the optical fibre sensor has been chosen such that it is longer than the length of one unit cell of the satin weave architecture $(7.4 \mathrm{~mm})$. The read-outs of the optical fibre sensor give the minimum and maximum local strains that occur along the length of the Bragg grating.
\end{abstract}

\section{INTRODUCTION}

In order to understand the complex structural deformation behavior of a textile reinforced composite, experimental local strain and local damage analysis prove to be valuable techniques. In the perspective of the experimental local strain analysis, due to the heterogeneity of textile composites, classical electrical resistance strain gauges do not have adequate spatial resolution and only a full-field strain measuring technique with high spatial resolution and strain sensitivity can be applied to determine local surface strain profiles (Nicoletto et al., in press). The local strain measurements obtained from the full-field method will provide a qualitative as well as quantitative understanding of the maximum and minimum values of local strains, and their corresponding locations on the laminate traction free surface. However, there are few publications (Lee et al., 2004; Potluri et al., in press; Lomov et al., 2008) that have studied the effect of adjacent layers in the laminate on the local strain behavior. In addition, the studies mentioned above suggested that the surface strain field is a representative strain field for all layers which may not be a reliable assumption. As adjacent layers try to suppress the yarn undulation effects of the laminate inner layers, they cause the local strain profile / gradient inside the laminate to vary considerably compared to the surface layers (Ivanov et al., in press; Ivanov, 2009). Moreover, it is important to understand the maximum and minimum strain locations due to the fact that these locations indicate the probable damage initiation zones (Potluri et al., in press; Summerscales \& Russell, 2004; Littell and Binienda, 2009).

In the above drawn guidelines, in order to quantify the local strains on the surface as well as the laminate inner layers, the following experimental approach is employed during the tensile test of the composite specimen: a) the local strain on the composite surface is quantified using surface mounted fibre optic sensors (FBG - Fibre Bragg Grating); b) embedded fibre optic sensors were used to obtain the maximum and minimum local longitudinal strain values inside the laminate. Surface mounted FBGs provided the quantitative local strain values. For the quantification of local strains inside the laminate, the only possible experimental technique of fibre optic sensors is employed. Based on the comparison of the local strain values at different locations, an attempt was made to understand the "shadowing" effects on the 
local strain behavior caused by the interaction of differently placed plies. Moreover, the large amount of available experimental data helps in the development of an accurate unit cell computational model for evaluating the local strain profiles using meso-FE simulations.

\section{EXPERIMENTAL PROCEDURE}

\subsection{Material and test method}

The material under study is a thermo-plastic 5harness satin weave composite (CD0286 supplied by 'Ten Cate'), which has T300 JB carbon fibres as a reinforcement and PPS as a matrix. The composite laminate is manufactured using the press forming technique, which has eight layers of satin weave fabric with 50:50 weight ratios in the warp and weft directions.

Tensile tests on the composite specimens were performed using a standard Instron machine (Instron 4505 , test speed $1 \mathrm{~mm} / \mathrm{min}$ ). Composite samples used for the tensile tests were prepared according to the ASTM standard (width $25 \mathrm{~mm}$, gauge length 170 $\mathrm{mm}$, thickness $2.5 \mathrm{~mm}$ ), with the end tabs of the same material (Carbon-PPS). The local strains during the tension test on the composite surface are measured using surface mounted optical fibre sensors. In addition, the local strains inside the laminate were measured using Fibre Optic Sensors.

\subsection{Strain measurement using fibre Bragg grating sensors}

Fibre optic sensors with fibre Bragg gratings (FBGs) are commonly used for local strain sensing, which makes them the optical counterpart to electrical strain gauges. The working principle of the Bragg grating within the optical fibre is explained in Figure 1 (Parlevliet, 2010). When a broadband light with a wide range of wavelengths travels through the optical fibre core containing a Bragg grating, a narrow band of wave length is reflected $\left(\lambda_{\text {ref }}\right)$, while the other wavelengths pass through the grating $\left(\lambda_{\text {trans }}\right)$. The central wavelength of the reflected light is called the Bragg wavelength $\lambda_{B}$ and this is shown in Figure 2.

In detail, an FBG is a sensitive element written in the core of an optical fibre. The refractive index changes periodically along a definite length in the fibre, acting as a reflector for a specific wavelength (Figure 2). The reflection spectrum is centred on the Bragg-wavelength $\lambda_{B}$ which is given by the condition $\lambda_{B}=2 n_{\text {eff }} \Lambda_{F B G}$, in which $n_{\text {eff }}$ is the effective refractive index of the core of the optical fibre and $\Lambda_{\text {FBG }}$ is the period of the grating. An FBG has typical dimensions of a few millimetres in length and a diameter of about $0.2 \mathrm{~mm}$.
In this study, high strength coated FBGs i.e. DTG ${ }^{\circledR}$ s (Draw Tower Gratings) were used. They can withstand high strain levels and have been found to be suitable for embedding in thermoplastic composites (De Baere et al., 2007).

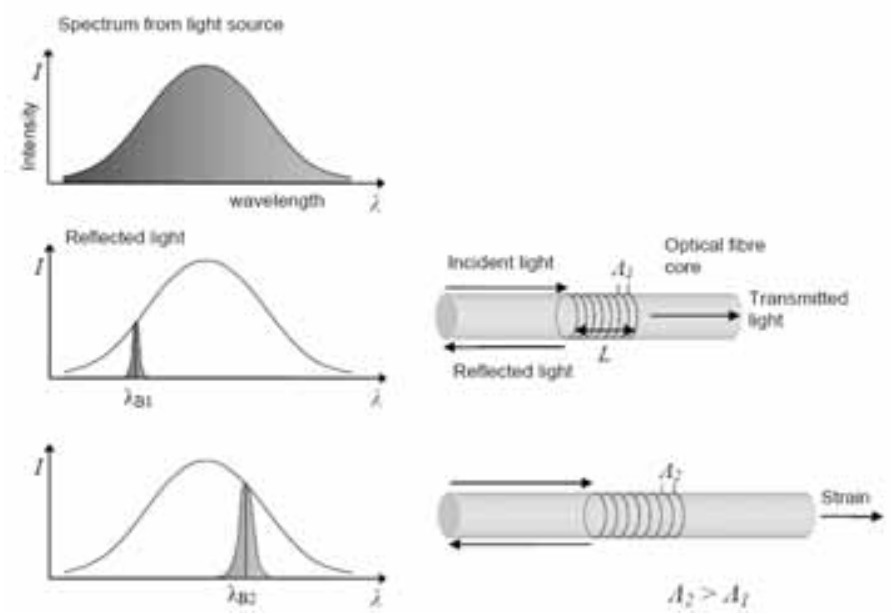

Figure 1. Schematic view of working principle of a fibre Bragg grating in a optical fibre (Parlevliet, 2010).
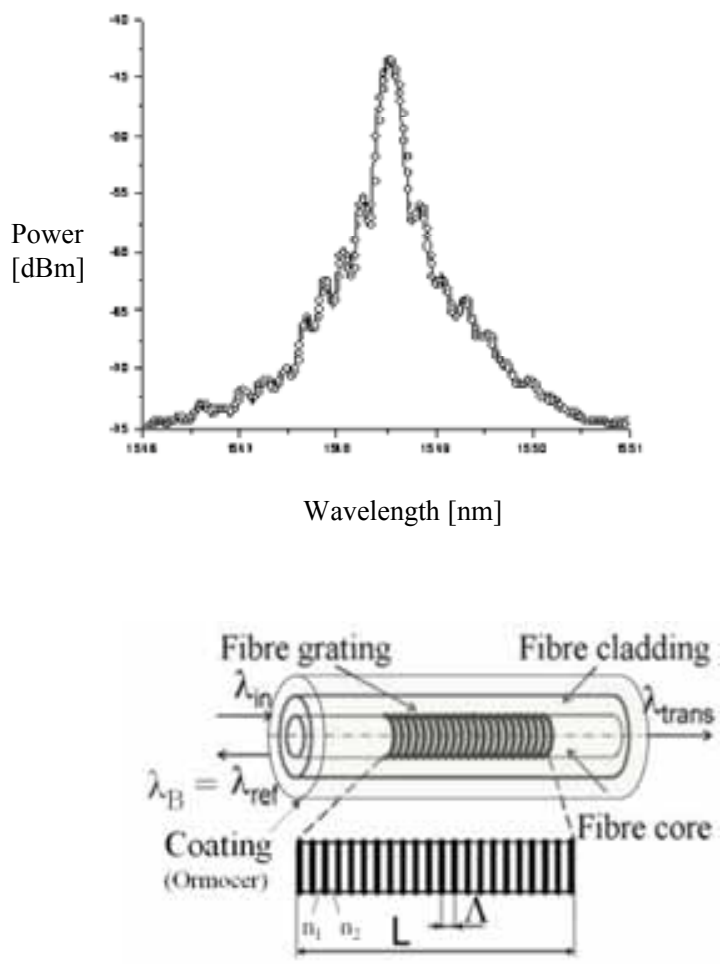

Figure 2. Typical reflected Bragg spectrum and FBG sensor in the fibre core.

The approach employed with regard to the use of fibre optic sensors in the current study is twofold: 1) FBGs were being mounted on the surface of the test specimens to measure local surface strains (Figure $3)$; 2) in order to capture the interior strains around the sensor, FBGs were directly embedded between layers of satin weave fabric (Figure 4). The latter procedure was as follows: the layers of carbon/PPS semi-preg were stacked in the mould. In the midplane of the laminate to be formed, the optical fibre sensors were laid out and fixed to the semi- 
preg with an ultrasonic welding torch. Next the stack of semi-pregs was compression moulded to consolidate the thermoplastic laminate.

In both approaches we have used sensors with a length of $8 \mathrm{~mm}$ (covering the complete length of one unit cell, i.e. $7.4 \mathrm{~mm}$ ). All sensors were connected to a commercial Bragg interrogator from FOS\&S (FBG-SCAN 608) with a depolarized optical source.
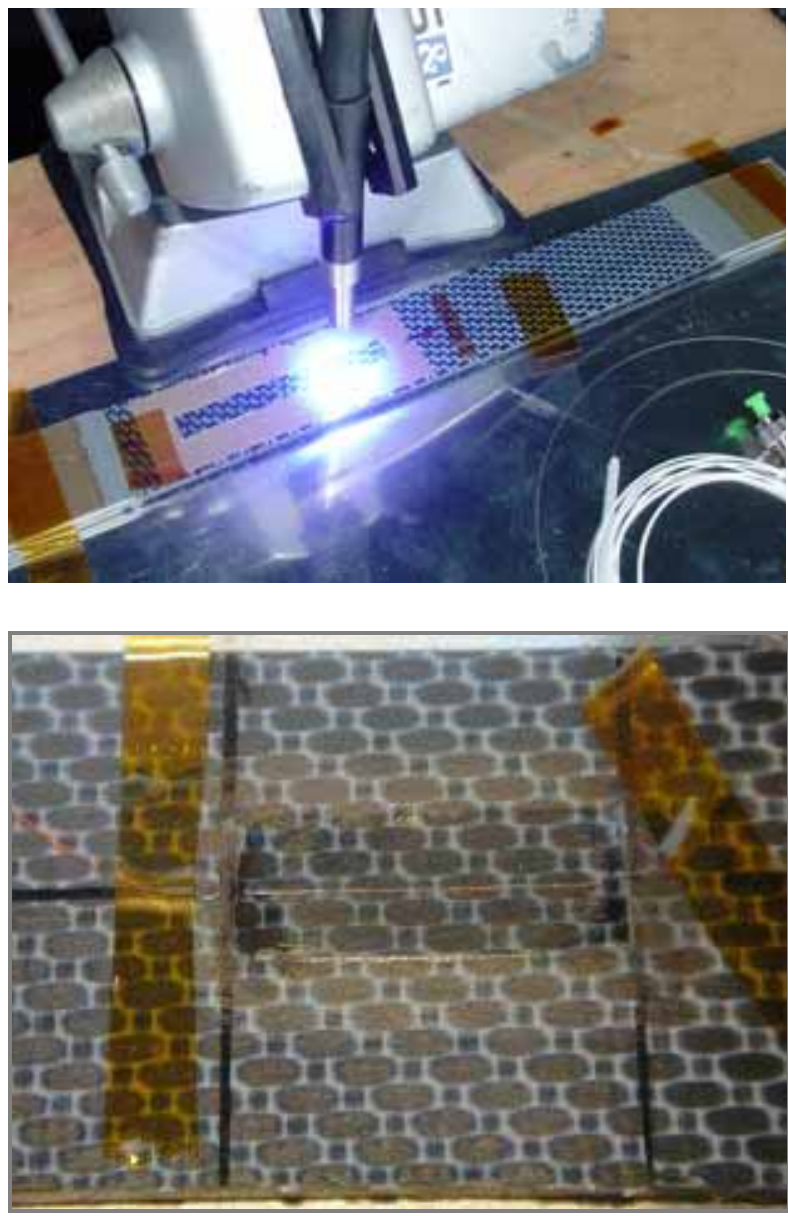

Figure 3. Surface mounted optical fibre sensors; a) glueing of the optical fibre on the test coupon using UV technique; b) mounted optical fibre on the composite surface.
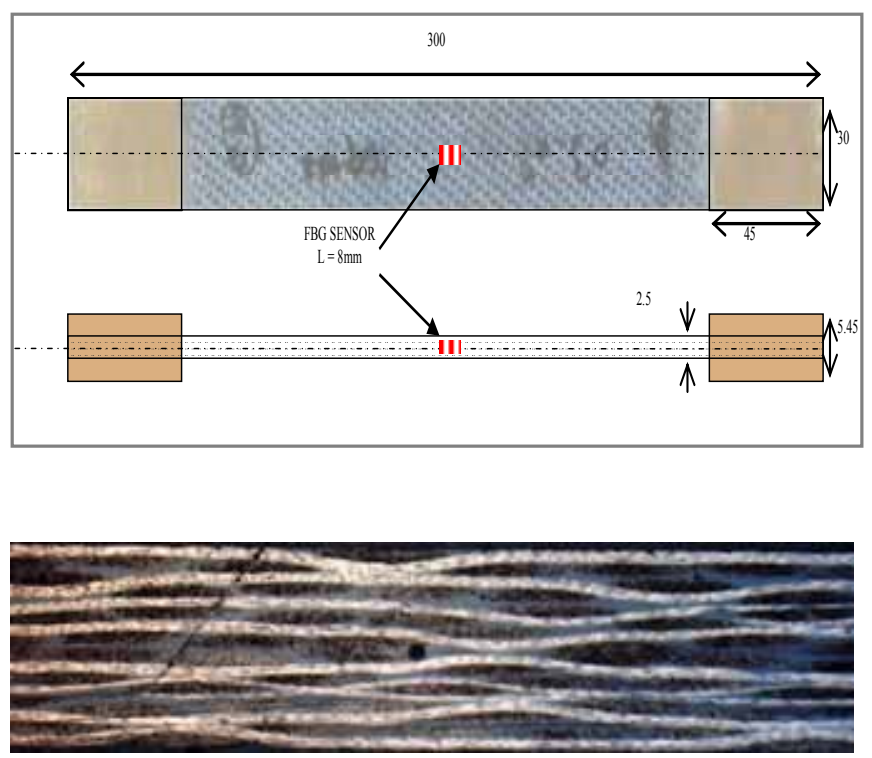

Figure 4. Embedded optical fibre sensors; a) top view; b) side view.

Both the Bragg interrogator and the test bench were linked to a PC and all read-out parameters (force, strain, Bragg spectra) were integrated in one software. During stepwise loading (quasi-static testing) of the composite specimens we were able to capture the response spectra from the composite at each load step.

The full spectral response of the FBG sensors and thus the complete Bragg peak deformation was examined for strain analysis. Peak distortion induced by the local non-uniform strain along the grating resulted in multiple small Bragg peaks (Figure 2). By converting the individual Bragg peak shifts into strain it is possible to calculate the local longitudinal maximum and minimum strain values along the grating length $(8 \mathrm{~mm})$.

Figure 5 shows the experimental set-up for the quasi-static tensile tests.

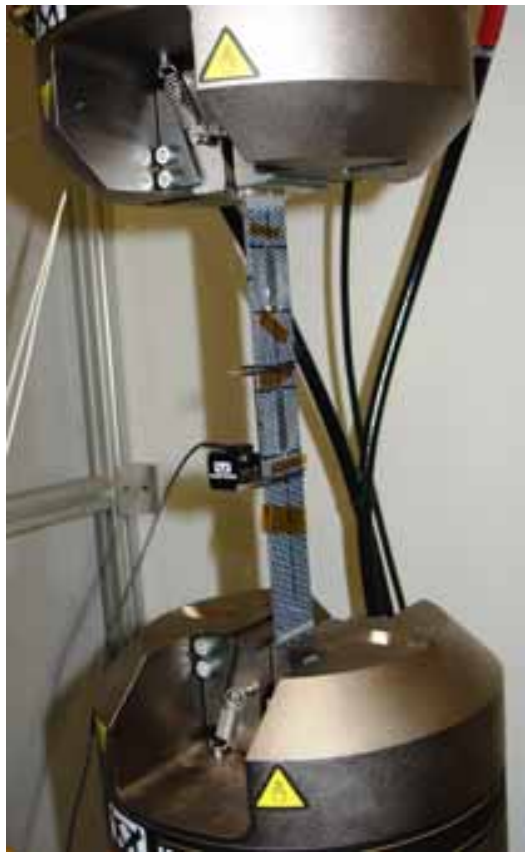

Figure 5. Experimental tensile test set up for measuring the local strains in the composite inner layers using FBGs.

\section{EXPERIMENTAL RESULTS}

\subsection{Surface strain measurement using FBGs}

Three test coupons were prepared with each specimen having two surface bonded $80 \mu \mathrm{m}$ DTG $^{\circledR}$ (high strength FBGs) sensors positioned in the central axis along the load carrying warp yarns (red line in Figure 4). The $80 \mu \mathrm{m}$ diameter sensors have a total diameter with ORMOCER $^{\circledR}$ coating of approximately $140 \mu \mathrm{m}$ and an FBG length of $8 \mathrm{~mm}$. A special method of the company FOS\&S has been employed to fix the sensors in a controlled manner using a UV adhesive and a special sensor pad 
(patent WO2009106576(A1)) as shown in Figure 3(a).

The goal is to measure the local longitudinal strain values (along a unit cell) during the tensile test. In addition to that, the surface strain values were compared with the strain values obtained from the embedded sensors to obtain the influence of the 'shadowing' effects caused by the internal yarn shifting (nesting) on the local strain values.

\subsection{Strain measurement in the laminate inner layers using FBGs}

In addition to the surface bonded sensors, the three test coupons mentioned above contain an embedded $125 \mu \mathrm{m} \mathrm{DTG}^{\circledR}$ sensor placed in the central plane of the composite laminate (Figure 4). The $125 \mu \mathrm{m}$ diameter sensors have a total diameter with ORMOCER ${ }^{\circledR}$ coating of approximately $190 \mu \mathrm{m}$ and an FBG length of $8 \mathrm{~mm}$.

It should be emphasized that the embedded sensor is surrounded by matrix material, yarns and warp and weft yarn cross-over points inducing a more complex strain field compared to the surface mounted sensors. It is therefore important to distinguish the transversal effects on the longitudinal strain measuring capabilities of the optical fibres. Previous research of Voet et al. (2008) has shown that transversal strains cause: i) optical birefringence effects in the core of the optical fibre; ii) nonuniform strain along the length of the FBG causing an in-fibre strain distribution. Both effects can be characterized by looking into the reflected spectrum of the Bragg peak. In addition, there is a strong correlation between the strain field acting on the embedded grating and the deformation of the reflected Bragg spectrum (Yiping Wang et al., 2006; Ling et al., 2007). However, further investigations on this issue have shown that the birefringence effect in the thermoplastic test specimens is mainly caused by the residual transverse stresses induced during the composite manufacturing process, and does not change significantly during the load process; i.e. from the beginning to the end of the loading process this effect stays more or less the same (below strain levels of $0.05 \%$ ). It can be stated that the Bragg peak deformations examined in this study were principally induced by longitudinal strain effects along the Bragg grating.

Finally, cross-sectional views of the composite laminate with an embedded optical fibre (Figure 6a, b) after the final failure of the composite showed that the optical fibre remained completely bonded to the host composite material, which ensured the correct and constant measuring of the local strains during the loading process.
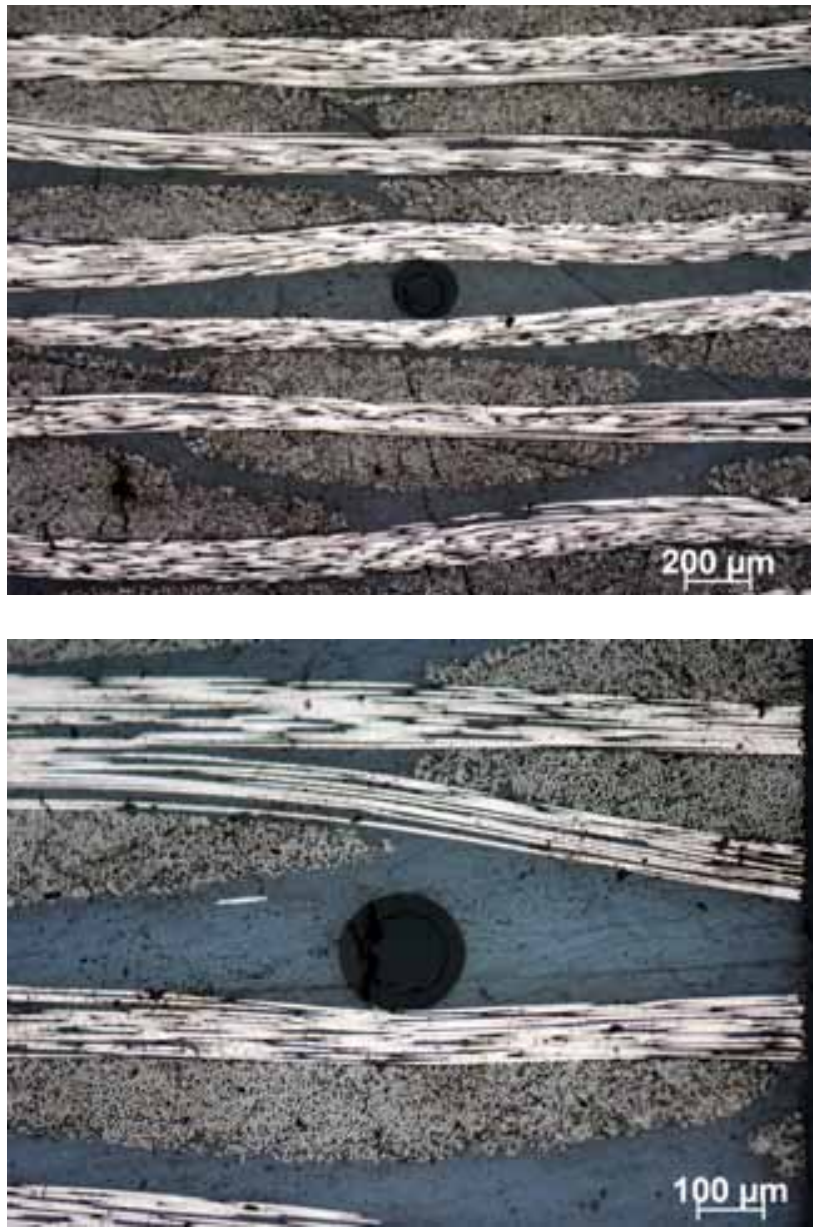

Figure 6. a, b) Cross-sectional views of the optical fibre embedded in the composite inner layers.

\subsection{Longitudinal strain calculation method}

In order to calculate the longitudinal local strain from the reflected spectrum of the fibre optic sensors, a logarithmic approach has been employed. To measure the strain independent of the environmental temperature fluctuations, a temperature compensating plate with a cantilever design is used. A DTG is fixed on the surface of the temperature compensating plate. It is then fixed on the test-coupon using some adhesive tape. The cantilever design ensures that no strain is applied on the temperature compensating plate, hence only thermal strain fluctuations are measured by the surface mounted DTG. With the above procedure, the strain induced in the composite specimen is calculated using the initial reference spectrum compared to the deformed spectrum under the applied tensile load. Moreover, for the strain formulation, temperature terms are also added as shown in the below equation.

$$
\Delta \varepsilon=\frac{\left(\ln \frac{\lambda_{\varepsilon}}{\lambda_{\varepsilon, 0}}-\ln \frac{\lambda_{T}}{\lambda_{T, 0}}\right)}{S_{\varepsilon}}=\frac{\left(\ln \frac{\lambda_{\varepsilon}}{\lambda_{\varepsilon, 0}}-\ln \frac{\lambda_{T}}{\lambda_{T, 0}}\right)}{0.777}
$$


The above equation is used to calculate the temperature compensated strain, where $\lambda_{\varepsilon, 0}$ and $\lambda_{T, 0}$ are the wavelength at the start of the measurement of the strain sensor and the temperature sensor, respectively, $\lambda_{s}$ and $\lambda_{T}$ are the respective measured wavelengths after applying the tensile load. Finally, the term $S_{\varepsilon}$ represents the calibrated strain gauge factor (Voet et al., 2008). Using the above mentioned experimental techniques, the obtained local strain values (surface, inside the laminate) are discussed in the following sections.

\subsection{Analysis of the experimental local strain profiles}

In order to calculate the local strain in the composite inner layers, the following approach is employed. Based on experimental acoustic emission and microscopic analysis of the same composite material, it has been shown previously by the authors (Daggumati et al., 2010a,b) that the damage initiation in different layers of the satin weave composite is a sequential process. Damage initiation began in the laminate inner layers at $0.2 \%$, followed by damage initiation on the surface layers at around $0.6 \%$ of the global tensile strain. In order to avoid the complexities posed by the damage initiation and propagation on the strain measuring capabilities of the optical fibre, the comparison of local strain between the traction free surface to the laminate inner layers is accomplished in the elastic range.

Figure 7a shows the embedded optical fibre response at various applied global tensile strain levels. Along with the thermal residual strains, the yarn crimp in the satin weave composite produces a deformed spectrum of the embedded sensor, without the application of any external structural load (dotted spectrum in 7a. Subsequently, application of the external tensile load produces the deformed spectra. Using equation 1 , the local strains in the composite inner layers as well as on the traction free surface (Figure $7(\mathrm{a}, \mathrm{b})$ ) are computed at different average tensile strains and listed in Table 1.

To correlate the minimum and maximum strain readouts from the optical fibre sensors with the geometrical locations inside the carbon/PPS unit cell, the numerical simulations of the unit cell have been used (Daggumati et al., 2010a;2010b). Those simulations show that the minimum longitudinal strain always occurs in the matrix pockets and the maximum longitudinal strain occurs in the yarn crimp region, where the weft bundle is crossing over the warp yarn. When the warp yarn is stretched under tension loading, the warp yarn is bent and local strain is high.
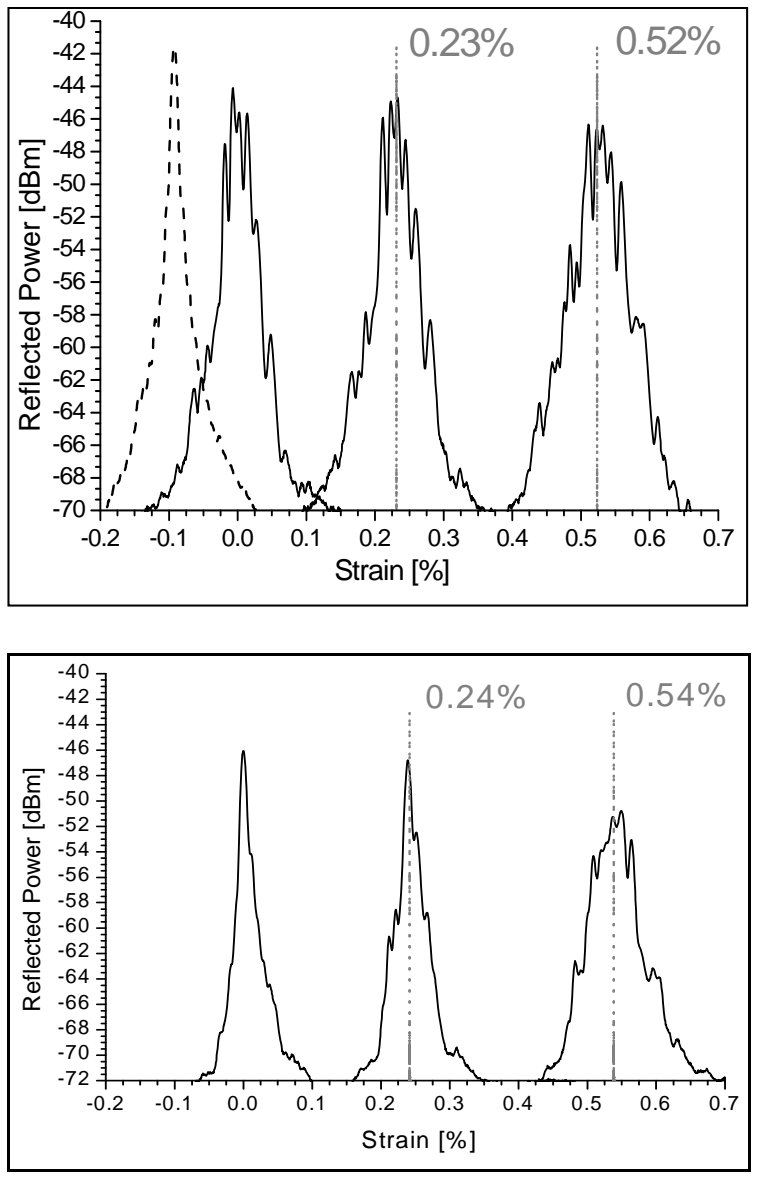

Figure 7. Optical fiber response spectrum at various strain levels: a) laminate inner layers; b) on the laminate traction free surface.

From Table 1 it is evident that, for the applied average tensile strain at around $0.2 \%$ and $0.5 \%$, embedded sensors detect the maximum local strain at around 0.25 and $0.55 \%$ respectively.

Table 1. Comparison of the local strain values - DIC vs. FBGs.

\begin{tabular}{|l|l|c|c|}
\hline & Average strain & $\begin{array}{l}\text { Local strain at } \\
\text { the yorn } \\
\text { crimp }(\text { weft }), \%\end{array}$ & $\begin{array}{l}\text { Local strain in } \\
\text { the matrix } \%\end{array}$ \\
\hline FBGs -inside & $(0.2 \pm 0.01) \%$ & 0.25 & 0.16 \\
\hline FBGs -inside & $(0.5 \pm 0.01) \%$ & $0.55-0.57$ & $0.13-0.45$ \\
\hline FBGs - surface & $(0.5 \pm 0.01) \%$ & $0.56-0.57$ & $0.12-0.45$ \\
\hline
\end{tabular}

The obtained local strain values at different locations of the laminate show that the effect of the internal yarn shifting (nesting) is negligible on the local longitudinal strain behaviour of the satin weave composite. The obtained local strain behaviour in the plies of a satin weave is in correlation to the observation made by Duplessis Kergomard et al. (2010) on the intra-yarn damage analysis of a 4-harness satin weave composite. According to Duplessis Kergomard et al. (2010), the local stress hence the local strain is affected mainly by the position of the ply in a weave structure, and 
the variability in the micro- and meso-structure. However, similar local strain behavior at various positions of the laminate suggests that the effect of internal yarn nesting is negligible on the local longitudinal strain values compared to the local strains induced at the yarn crimp region, by straightening of the weft yarns.

\section{SUMMARY}

Comprehensive experimental local strain analysis is performed on a thermoplastic carbon-PPS 5-harness satin weave composite under uni-axial static tensile load. For the analysis of local strain on the laminate surface, surface mounted fibre optic sensors were used. In order to detect the local strain inside the laminate, fibre optic sensors were embedded into the laminate during the composite manufacturing process. The detailed summary of the experimental results is presented here.

- The maximum local strain on the laminate surface occurs at the centre of the weft yarn at the yarn crimp location, while the minimum strain occurs in the resin pockets adjacent to the yarn crimp locations.

- Along the straight portion of the warp yarn, the local strain is almost equal to the average strain applied to the laminate.

- At the applied average tensile strain of $0.5 \%$, the local maximum and minimum strain values obtained from the surface mounted and embedded optical fibres show almost the same strain, indicating that the 'shadowing' effects are negligible on the local longitudinal strain behaviour in the plies of a satin weave composite.

The qualitative strain locations observed in the current study are compared to the maximum and minimum strain locations that have been observed in other studies on the local strain analysis of plain and twill weave composites under uni-axial static tensile load. From the above observations, it might be concluded that for all 2D woven composites, the local in-plane strain components such as longitudinal, transverse and shear strains show similar behaviour on the composite traction free surface. Finally, consistency in the local strain values obtained from the different experimental techniques demonstrates the accuracy of the used experimental procedures.

\section{REFERENCES}

Daggumati, S., et al., Local damage in a 5-harness satin weave composite under static tension: Part II - Meso-FE modelling. Composites Science and Technology. 2010, 70(13): p. 1934-1941.

Daggumati, S., et al., Local damage in a 5-harness satin weave composite under static tension: Part I - Experimental analysis. Composites Science and Technology. 2010, 70(13): p. 1926-1933.

De Baere, I. et al., Strain Monitoring in Thermoplastic Composites with Optical Fiber Sensors: Embedding Process, Visualization with Micro-tomography, and Fatigue Results. Journal of Thermoplastic Composite Materials, 2007.

Duplessis Kergomard, Y., et al., Intralaminar and interlaminar damage in quasi-unidirectional stratified composite structures: Experimental analysis. Composites Science and Technology. 2010, 70(10): p. 1504-1512.

Ivanov, D., et al., Strain mapping analysis of textile composites. Optics and Lasers in Engineering. In Press, Corrected Proof.

Ivanov, d.S., Damage analysis in textile composites. $\mathrm{PhD}$ Thesis; KU Leuven - Faculty of Engineering, May 2009.

Lee, J.-R., et al., Digital phase-shifting grating shearography for experimental analysis of fabric composites under tension. Composites Part A: Applied Science and Manufacturing, 2004. 35(7-8): p. 849-859.

Ling, H.-Y., et al., Characterization of dynamic strain measurement using reflection spectrum from a fiber Bragg grating. Optics Communications, 2007. 270(1): p. 25-30.

Littell, J.D. and W.K. Binienda, Characterization of Damage in Triaxial Braid Composites Under Tensile Loading. NASA/TM-2009-215645, May 2009.

Lomov, S.V., et al., Full-field strain measurements for validation of meso-FE analysis of textile composites. Composites Part A: Applied Science and Manufacturing, 2008. 39(8): p. 1218-1231.

Nicoletto, G., G. Anzelotti, and E. Riva, Mesoscopic strain fields in woven composites: Experiments vs. finite element modeling. Optics and Lasers in Engineering. In Press, Corrected Proof.

Parlevliet, P.P., Residual Strains in Thick Thermoplastic Composites - an Experimental Approach. PhD Thesis; Delft University of Technology, 2010.

Potluri, P., et al., Meso-scale Strain Mapping in UD Woven Composites. Composites Part A: Applied Science and Manufacturing. In Press, Accepted Manuscript.

Summerscales, J. and P.M. Russel, Observations on the fibre distribution and fibre strain in a woven fabric reinforcement. Advanced composite letters 2004. 23 No.3, 2004.

Voet, E. et al., High strain measurements during fatigue cycleing in fibre reinforced thermoplastic composites using imbedded draw tower fibre brag grating sensors ECCM13 Conference procedings, 2008.

YipingWang, et al., Characterization of a high birefringence fibre Bragg grating sensor subjected to non-homogeneous transverse strain fields. Meas. Sci. Technol. 17 939-942 2006. 